\title{
artigo
}

Graça, V. V., Rodrigues, I. L. A., Palmeira, I. P., Bezerra, D. F., Martins, A. C. G. S., Sá, A. M. M.

Perfil sociodemográfico e epidemiológico da doença de chagas aguda em um município amazônico

\section{Perfil sociodemográfico e epidemiológico da doença de chagas aguda em um município amazônico}

\author{
Socio-demographic and epidemiological profile of acute chagas disease in an amazon municipality \\ Perfil socio demográfico y epidemiológico de la enfermedad aguda de chagas en un municipio amazónico
}

\begin{abstract}
RESUMO
Objetivo: Identificar o perfil sóciodemográfico da Doença de Chagas Aguda (DCA) no município de Abaetetuba-Pará. Método: Estudo descritivo, analítico, transversal, com abordagem quantitativa, realizado em Abaetetuba-PA. Participaram 20 pacientes com DCA acompanhados em ambulatório municipal. Para a coleta de dados utilizou-se um formulário com perguntas abertas e fechadas. Resultados: Os pacientes estão na faixa etária entre 20 e 39 anos $(40 \%)$, sexo masculino $(50 \%)$, união estável $(35 \%)$, solteiros (35\%), baixa escolaridade $(55 \%)$, não possuem renda $(60 \%)$, declaram ter alguma religião com participação ativa $(90 \%)$, católicos $(50 \%)$, realizando tratamento $(55 \%)$, não estavam tratando em decorrência de: reações adversas ao tratamento com benznidazol; ou que ainda iriam iniciar o tratamento; ou haviam concluído recentemente, e apenas um participante evoluiu com complicação. Conclusão: Evidenciou-se as vulnerabilidades dos participantes e permitiu a elaboração de estratégias preventivas, uma vez que a baixa renda e escolaridade estão relacionadas com a maior prevalência da DCA.
\end{abstract}

DESCRITORES: Doença de Chagas Aguda; Epidemiologia; Amazônia.

\section{ABSTRACT}

Objective: To identify the sociodemographic profile of Acute Chagas Disease (ACD) in the city of Abaetetuba-Pará. Method: Descriptive, analytical, cross-sectional study with a quantitative approach, carried out in Abaetetuba-PA. Twenty patients with ACD followed at a municipal clinic participated. For data collection, a form with open and closed questions was used. Results: Patients are aged between 20 and 39 years $(40 \%)$, male $(50 \%)$, in a stable relationship (35\%), single (35\%), low education (55\%), have no income $(60 \%)$ declared to have a religion with active participation (90\%), Catholics $(50 \%)$, undergoing treatment $(55 \%)$, were not receiving treatment due to: adverse reactions to treatment with benznidazole; or that they were about to start treatment; or had recently completed, and only one participant evolved with complications. Conclusion: The vulnerabilities of the participants were highlighted and allowed the development of preventive strategies, since low income and education are related to the higher prevalence of $A C D$.

DESCRIPTORS: Acute Chagas Disease; Epidemiology; Amazon.

\section{RESUMEN}

Objetivo: Identificar el perfil sociodemográfico de la Enfermedad de Chagas Aguda (ECA) en la ciudad de Abaetetuba-Pará. Método: Estudio descriptivo, analítico, transversal con abordaje cuantitativo, realizado en Abaetetuba-PA. Participaron veinte pacientes con ECA seguidos en una clínica municipal. Para la recolección de datos se utilizó un formulario con preguntas abiertas y cerradas. Resultados: Los pacientes tienen entre 20 y 39 años $(40 \%)$, hombres $(50 \%)$, en relación estable $(35 \%)$, solteros $(35 \%)$, baja escolaridad $(55 \%)$, no tienen ingresos $(60 \%)$, declararon tener una religión con participación activa $(90 \%)$, católicos $(50 \%)$, en tratamiento $(55 \%)$, no recibían tratamiento debido a: reacciones adversas al tratamiento con benznidazol; o que estaban a punto de comenzar el tratamiento; o había completado recientemente, y solo un participante evolucionó con complicaciones. Conclusión: Se resaltaron las vulnerabilidades de los participantes y se permitió el desarrollo de estrategias preventivas, ya que los bajos ingresos y la educación se relacionan con la mayor prevalencia de ECA.

DESCRIPTORES: Enfermedad de Chagas aguda; Epidemiología; Amazonas.

RECEBIDO EM: 18/10/2021 APROVADO EM: 17/01/2022

\section{Victor Viana da Graça}

Enfermeiro. Doutorando em Biologia Parasitária da Amazônia pela Universidade do Estado do Pará (UEPA).

ORCID: 0000-0002-6144-4241

\section{Ivaneide Leal Ataíde Rodrigues}

Enfermeira. Doutora em Enfermagem. Docente do PPG Mestrado em Enfermagem da Universidade do Estado do Pará (UEPA). ORCID: 0000-0001-9968-9546 


\section{lací Proença Palmeira}

Enfermeira. Doutora em Enfermagem. Docente do PPG Mestrado em Enfermagem da Universidade do Estado do Pará (UEPA) ORCID: 0000-0001-9659-3565

\section{Daniele Ferreira Bezerra}

Acadêmica de Enfermagem pela Universidade da Amazônia (UNAMA).

ORCID: 0000-0002-0068-7741

\section{Ana Caroline Guedes Souza Martins}

Enfermeira. Doutoranda em Pesquisa Clínica em Doenças Infecciosas pelo Instituto Nacional de Infectologia (INI-FIOCRUZ). ORCID: 0000-0001-7185-8520

\section{Antônia Margareth Moita Sá}

Enfermeira. Doutora em Enfermagem. Docente do PPG Mestrado em Enfermagem da Universidade do Estado do Pará (UEPA). ORCID: 0000-0002-2053-5622

\section{INTRODUÇÃO}

A Doença de Chagas (DC) é uma antropozoonose causada pelo protozoário flagelado, o Trypanosoma cruzi tendo como vetor o inseto da espécie de triatomíneo, popularmente conhecido como "barbeiro". Na ocorrência da doença, observam-se duas fases clínicas: uma aguda, que pode ou não ser identificada, podendo evoluir para uma fase crônica caso não seja tratada com medicação específica. No Brasil, devido à transmissão vetorial domiciliar ocorrida no passado e hoje interrompida, predominam os casos crônicos. Estima-se que existam entre dois e três milhões de indivíduos infectados ${ }^{1}$.

No entanto, nos últimos anos, a ocorrência de Doença de Chagas Aguda (DCA) tem sido observada em diferentes estados, em especial na região da Amazônia Legal, principalmente, em decorrência da transmissão oral, com a ocorrência de surtos relacionados à ingestão de alimentos contaminados (caldo de cana, açaí, bacaba, entre outros) e casos isolados por transmissão vetorial extradomiciliar ${ }^{1}$.

$\mathrm{Na}$ DCA predomina o parasito circulante na corrente sanguínea, em quantidades expressivas. Tendo a febre persistente como a manifestação mais característica, inicialmente elevada $\left(38,5\right.$ a $\left.39^{\circ} \mathrm{C}\right)$, como possíveis picos vespertinos ocasionais. As manifestações de síndrome febril podem persistir por até 12 semanas. Esta fase inicial, mesmo não tratada nem diagnosticada, pode evoluir com desaparecimento espon- tâneo da febre e da maior parte das outras manifestações clínicas, evoluindo para a fase crônica. Em alguns casos, com quadro clínico mais grave, pode evoluir para óbito ${ }^{2}$.

É considerado caso suspeito de DCA a pessoa que tenha ingerido alimento suspeito de contaminação por T. cruzi ou visitado área de ocorrência de triatomíneos e apresente febre prolongada (superior a 7 dias), acompanhado de pelo menos um dos seguintes sinais: edema de face ou de membros, exantema, adenomegalia, hepatomegalia, esplenomegalia, cardiopatia aguda (taquicardia, sinais de insuficiência cardíaca), manifestações hemorrágicas, sinal de Romaña ou chagoma de inoculação ${ }^{3}$.

A droga disponível para o tratamento específico da DC é o benznidazol apresentado na forma de comprimidos de $100 \mathrm{mg}$. $\mathrm{Na}$ fase aguda, o tratamento deve ser realizado em todos os casos e o mais célere possível após a confirmação diagnóstica. Deve ser usado em duas ou três tomadas diárias por via oral, durante 60 dias, e a dose varia de acordo com a idade e o peso do paciente 2 .

No período de 2010 a 2017 o Brasil teve 1.866 casos confirmados de DCA, sendo que 1.102 foram no estado do Pará, que equivale a 59,05\% dos casos. Destes, 348 casos $(31,57 \%)$ eram oriundos da região de Saúde de Tocantins e 181 casos pertenciam ao município de Abaetetuba, município com maior número de casos confirmados da doença, ou seja, $16,42 \%$ dos casos do Pará e $52 \%$ dos casos confirmados na regional de saúde do Tocantins. Observada a distribuição de casos de DCA para cada ano analisado, a maior ocorrência se deu em 2016, com 282 casos confirmados (25,58\%), o que caracterizou aquele ano como o de maior incidência da doença. O ano de 2011 foi o de menor incidência, com 24 casos confirmados $(2,17 \%)^{4}$.

Desde 2012, a DC representa uma condição infecciosa (com fase aguda ou crônica) e compõe o conjunto de doenças negligenciadas, classificadas como doenças associadas à situação de pobreza e precárias condições de vida e às iniquidades em saúde, conforme a Organização Mundial de Saúde (OMS) .

Diante desse contexto, esta pesquisa teve como objetivo identificar o perfil sóciodemográfico da Doença de Chagas Aguda no município de Abaetetuba-Pará

\section{MÉTODO}

Pesquisa com delineamento descritivo, analítico e transversal, com abordagem quantitativa, realizada no município de Abaetetuba-PA, Brasil, pertencente à região de saúde Tocantins, vinculado ao $6^{\circ}$ Centro Regional de Saúde-SESPA, que integra a Mesorregião Nordeste Paraense.

Participaram da pesquisa 20 pacientes com DCA que fazem acompanhamento em um ambulatório municipal. Para os critérios de inclusão: Pacientes com DCA, em acompanhamento no serviço de saúde municipal de Abaetetuba, de ambos os sexos e idade a partir de 12 anos. Para os critérios de exclusão: Não residentes do município de Abaetetuba-PA, pessoas com déficit 


\section{artigo}

Graça, V. V., Rodrigues, I. L. A., Palmeira, I. P., Bezerra, D. F., Martins, A. C. G. S., Sá, A. M. M.

Perfil sociodemográfico e epidemiológico da doença de chagas aguda em um município amazônico

cognitivo que impossibilitam responder aos questionamentos da entrevista.

Para a coleta de dados utilizou-se um formulário com perguntas abertas e fechadas, composto por: idade, sexo, escolaridade, estado civil, religião, profissão/ocupação, renda pessoal, tratamento e complicações da DC. Optou-se em realizar a entrevista nos domicílios para que não houvesse interferência na rotina do serviço de saúde e para que o paciente tivesse maior conforto para responder aos questionamentos.

Para a análise dos dados, as variáveis encontradas foram tabuladas em uso do Microsoft Excel 2010 para descrição de médias, desvio padrão e valores de porcentagem, assim como para elaboração de gráficos e tabelas.

A pesquisa respeitou a resolução nº66/12 do Conselho Nacional de Saúde que regulariza e normatiza a pesquisa envolvendo seres humanos. O projeto foi submetido ao Comitê de Ética em Pesquisa do Curso de Graduação em Enfermagem da Universidade do Estado do Pará-UEPA, aprovado sob o número do parecer 2.288.005, CAAE: 75435417.2 .0000 .5170 e obteve também a aprovação da Secretaria Municipal de Saúde.

\section{RESULTADOS}

Os resultados apontam que a maior frequência de idade foi na faixa etária entre 20 e 39 anos (40\%), com idade média de 33 anos, sendo a idade mínima 27 anos e a máxima de 38 anos (Gráfico 1). Logo em seguida as faixas etárias de 12 a 19 anos ( $n=6,50 \%$ com idade igual ou maior que 18 anos) e a de 40 a 59 anos ( $n=5)$, em que para primeira a idade média foi de 17 anos, sendo a idade mínima de 14 anos e a máxima 19 anos, já na segunda à idade média foi de 48 anos, com a idade mínima de 40 anos e a máxima 59 anos. Em se tratando de faixas etárias economicamente ativas, o estudo mostrou predominância nas faixas de 20 a 39 anos e de 40 a 59 anos, com 13 participantes (65\%).

Não houve predominância de sexo pois dos 20 participantes, $10(50 \%)$ eram do sexo masculino, e 10 (50\%) do feminino.

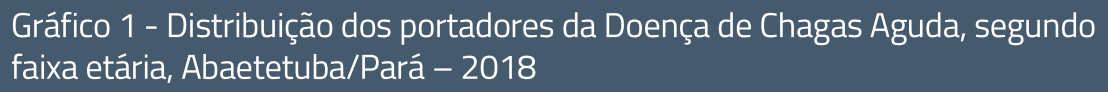

\section{FAIXA ETÁRIA}

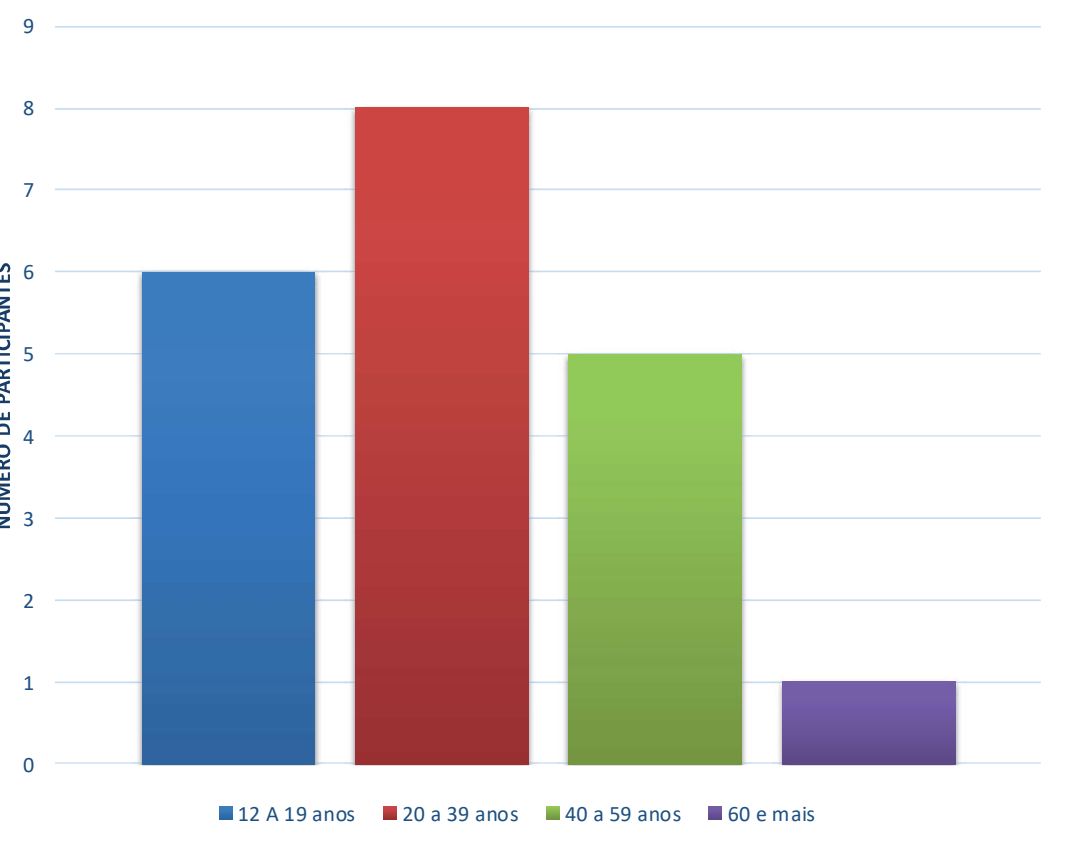

Fonte: elaborado pelo autor

Gráfico 2 - Distribuição dos portadores da Doença de Chagas Aguda, segundo escolaridade, Abaetetuba/Pará - 2018

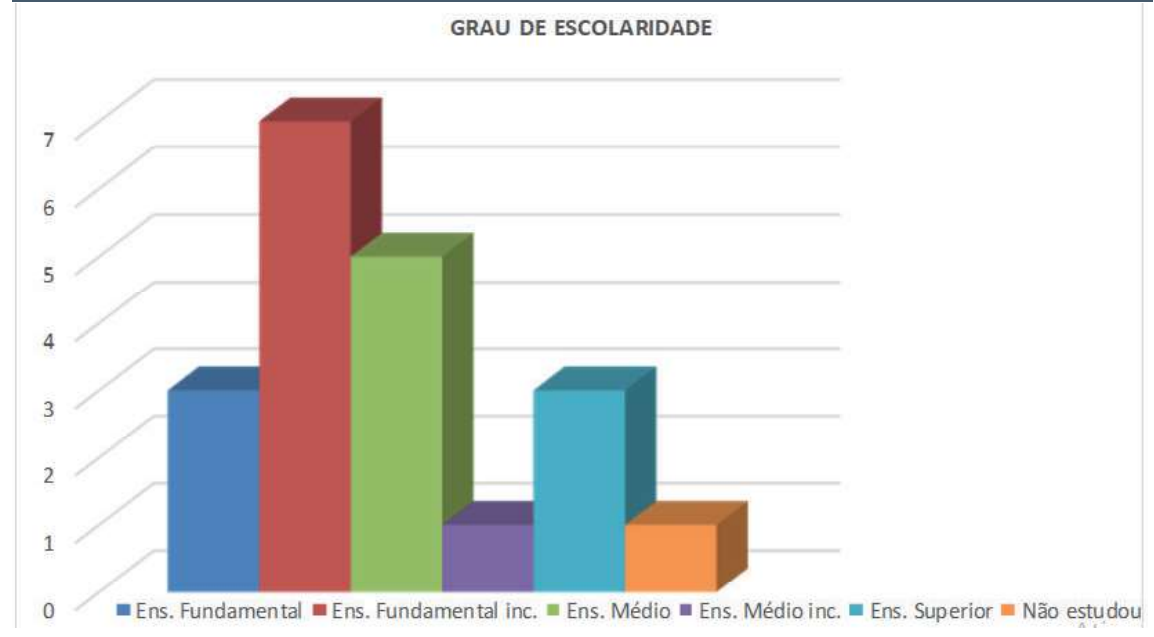

Fonte: elaborado pelo autor

Quanto ao estado civil, observou-se que a maioria vive em união estável $(7 ; 35 \%)$ e solteiros $(7 ; 35 \%)$. No que diz respeito à escolaridade e à renda, o maior número de casos ocorreu em indivíduos que não estudavam e com indivíduos com baixa escola- ridade $(11 ; 55 \%)$ com ensino fundamental incompleto e com ensino fundamental completo (Gráfico 2).

Constatou-se que 12 pacientes não possuem renda $(60 \%)$ e seis apresentam renda menor que um salário mínimo, em um total 
de 18 dos 20 participantes, evidenciando a vulnerabilidade social. Os participantes declaram ter alguma religião com participação ativa $(18 ; 90 \%)$, com destaque para a religião católica $(10 ; 50 \%), 35 \%$ Evangélicos, $10 \%$ sem religião e $5 \%$ Espírita. Quanto ao tratamento da DCA, $60 \%(n=12)$ estavam realizando o tratamento. Os demais, relataram que não estavam tratando em decorrência de alguns fatores, como: reações adversas ao tratamento com benznidazol; ou que ainda iriam iniciar o tratamento; ou haviam concluído recentemente. Sobre o aparecimento de complicação em decorrência da DCA entre os participantes, apenas um participante (5\%) evoluiu com complicação do tipo cardíaca.

\section{DISCUSSÃO}

Neste estudo a variável faixa etária obteve a média em 33 anos, divergindo do estudo de Vargas et al. ${ }^{6}$, que evidenciou média da faixa etária de 49 anos, moradores de zona rural e consumidores de caldo de cana.

A média de idade encontrada neste estudo pode ser explicada pela maior prevalência da DCA no estado do Pará, com transmissão oral predominando as causas, relacionados ao consumo do suco de açaí, considerado um alimento essencial na dieta da população da Região Norte e muito apreciado nos demais estados brasileiros e em outros países ${ }^{7}$. A epidemiologia da doença em Abaetetuba-PA está associada significativamente com a cultura alimentar do açaí produzido nas ilhas com a ocorrência dos casos agudos por transmissão oral na área urbana ${ }^{8}$.

Quanto a variável sexo, nesta pesquisa não houve predominância. Porém, em um estudo sobre o perfil dos pacientes com DCA no estado do Acre, acompanhados em ambulatório de serviços especializados, mostrou que a predominância em relação ao sexo masculino $(72,09 \%)$, relacionado a maior taxa de transmissão pela picada do barbeiro, ainda destacando que a predominância dos homens por conta do trabalho no campo e zona rural ${ }^{9}$.

Entretanto os resultados em relação ao gênero corroboram com estudos ${ }^{10}$, em que realizou uma análise espaço-temporal da DC no município de Barcarena-Pará, e evidenciou que não houve significância estatística em relação ao sexo, relacionado também pela alta prevalência da DC no estado do Pará, afetando igualmente independente do sexo.

Sobre o estado civil dos participantes, predominou solteiros, porém sem significância estatística. Sendo este resultado contrário em relação à pesquisa de Andrade ${ }^{11}$, que traçou o perfil epidemiológico de pacientes chagásicos em um hospital referência em Belém-PA, e destacou que $57 \%$ eram casados, seguido de $43 \%$ solteiros.

Estudos realizados em Minas Gerais e Pará corroboram com o presente estudo, com predominância do ensino fundamental incompleto $(38 \%)^{12}$. Desta maneira mostra-se que a DCA afeta predominantemente indivíduos com baixa escolaridade. Desta maneira, tanto no contexto Amazônico e quanto no Sudeste, a escolaridade se encontra semelhante ${ }^{11}$.

Quanto à renda, constatou-se que $60 \%$ correspondeu até um salário mínimo. Indo em concordância, uma pesquisa realizada no Ceará, com 89 pacientes chagásicos, atendidos em um serviço de atenção farmacêutica, evidenciaram que a renda de um salário mínimo predominou em $70 \%$, e relacionando a baixa escolaridade com a baixa renda, mostra que esses indivíduos possuem pouco acesso as práticas de promoção e educação em saúde. Sendo assim, justifica-se o acompanhamento dos pacientes em serviços especializados com o objetivo de oferecer melhor qualidade de vida, através da adesão ao tratamento e práticas preventivas para a ocorrência de novos casos naquela comunidade ${ }^{13}$.

Sobre a religião dos participantes, $50 \%$ referiram serem católicos. Já em um estudo realizado em Belém-PA, mostrou a predominância em relação a religião foi $57,14 \%$ de evangélicos e 42,85\% de católicos, sendo contrário aos resultados deste estudo?. A crença em uma religião e nas atividades religiosas é uma forma de manter-se ativo e esperançoso. Assim repercutindo na qualidade de vida na questão psicossocial, uma vez que essas atividades minimizam o risco de isolamento e promovem interação social ${ }^{14}$.

Abordou-se em relação ao tratamento, em que $60 \%$ referiram estar em tratamento. Um estudo que avaliou 19 pacientes com DCA em Fortaleza-CE constatou que 55\% não aderiu ao tratamento com o benznida$\mathrm{zol}^{15}$.

De acordo com o Protocolo Clínico e Diretrizes Terapêutica Doença de Chagas do Ministério da Saúde16, o benznidazol é medicamento mais utilizado. No entanto, apresenta uma taxa de $53 \%$ de eventos adversos: parestesias $(10,3 \%)$, artralgias $(8,1 \%)$, intolerância gastrointestinal $(13,3 \%)$, eventos dermatológicos como, alopecia $(0,9 \%)$, dermatites e rash cutâneo (30-44\%), comumente sem necessidade de interromper o tratamento. Entretanto alguns sintomas como parestesias (polineuropatia periférica), representam alto impacto na funcionalidade e qualidade de vida, persistindo por meses após a interrupção do tratamento. Também podem ocorrer complicações mais graves, como depressão da medula óssea com neutropenia, assim recomenda-se a realização do hemograma a cada três semanas para o monitoramento ${ }^{16}$.

Constata-se que a situação epidemiológica da DCA se assemelha com outras doenças endêmicas da região amazônica, a exemplo da Leishmaniose Visceral, costuma atingir indivíduos de baixo poder aquisitivo. Essa particularidade social pode interferir no estado de saúde da população pelas condições sanitárias precárias que vivem, em que as infecções e a desnutrição agravadas pelas doenças são fatores de mau prognóstico $^{17}$.

Neste estudo, quanto à presença de complicações relacionadas a DCA, $5 \%$ possui alguma complicação. A evolução clínica da fase aguda DC na maioria das vezes é benigno e os sinais e sintomas naturalmente desaparecem de forma espontânea em torno de 1 a 3 meses. Entretanto, a evolução fatal acontece em $<5 \%$ dos pacientes na fase aguda, quando contaminados por via vetorial clássica (picada do inseto triatomíneo), principalmente em pacientes que evoluem para insuficiência cardíaca refratária. Apesar disso, nos casos agudos ocasionados por contaminação por via oral, a doença 


\section{artigo}

Graça, V. V., Rodrigues, I. L. A., Palmeira, I. P., Bezerra, D. F., Martins, A. C. G. S., Sá, A. M. M.

Perfil sociodemográfico e epidemiológico da doença de chagas aguda em um município amazônico

aguda costuma ser mais grave e a mortalidade notada é mais alta. Isso possivelmente se deve à inoculação de alta carga parasitária e à capacidade de penetração por meio da mucosa gastrointestinal, muito permeável ao parasita, nessas situações ${ }^{18}$.

\section{CONCLUSÕES}

Através deste estudo foi possível alcançar o objetivo proposto através do conhecimento do perfil sóciodemográfico dos pacientes com DCA acompanhados em um ambulatório especializado em Abaetetuba-PA, o que evidenciou as vulnerabilidades dos participantes e permite a elaboração de estratégias preventivas, uma vez que, destaca-se a baixa renda e escolaridade relacionadas com a maior prevalência da DCA. Desta maneira espera-se que os resultados desta pesquisa promovam um melhor planejamento em prol da promoção e educação em saúde desta população, com o intuito de reduzir a morbimortalidade relacionada a DCA.

\section{REFERÊNCIAS}

1. Brasil. Ministério da Saúde. Guia de vigilância em saúde. 1st ed. Brasília: Secretaria de Vigilância em Saúde; 2017. 250 p. Disponivel em: http://bvsms.saude.gov.br/bvs/publicacoes/guia_vigilancia_ saude_volume_1.pdf Acesso em 24 ago 2019.

2. Dias JCP, Ramos AN, Luquetti, Gontijo ED, Alejandro, Torres RM, et al. II Consenso Brasileiro em Doença de Chagas, 2015. Epidemiol Serv Saúde. 2016 doi: http://www.iec.pa.gov.br/template doi_ess.php?doi=10.5123/S1679-49742016000500007\&scielo $=$ S2237-96222016000500007

3. Brasil M da S. Guia de vigilância em saúde. 1st ed. Brasília: Secretaria de Vigilância em Saúde; 2014. 814 p. Disponível em: www. saude.gov.br/svs Acesso em 24 ago 2019.

4. Brasil M da S. Guia de vigilância em saúde. 1st ed. Brasília: Secretaria de Vigilância em Saúde; 2017; 286 p. Disponivel em: www. saude.gov.br/bvs Acesso em 24 ago 2019.

5. Brasil. Ministério da Saúde. Doenças negligenciadas: estratégias do Ministério da Saúde. Rev Saúde Pública. 2010. doi: $\quad$ http://www.scielo.br/scielo.php?script=sci_arttext\&pid=S0034-89102010000100023\&lng=pt\&tlng=pt

6. Vargas A, Malta JMAS, da Costa VM, Cláudio LDG, Alves RV, Cordeiro $\mathrm{G}$ da $\mathrm{S}$, et al. Investigação de surto de doença de Chagas aguda na região extra-amazônica, Rio Grande do Norte, Brasil, 2016. Cad Saude Publica. 2018. 34(1):1-8.

7. Ferreira RTB, Branquinho MR, Leite PC. Transmissão oral da doença de Chagas pelo consumo de açaí: um desafio para a Vigilância Sanitária. Vig. Sanit. Debate, Rio de Janeiro, 2(4): 4-11, 2014.

8. Gonçalves ES. Mosaico de vulnerabilidades: açaí artesanal e risco da transmissão oral da doença de Chagas. Tese de Doutorado. Universidade de São Paulo. 2017.

9. Teixeira RB, Oliveira SMC de. Perfil de pacientes portadores de Doença de Chagas em Rio Branco, Acre, Brasil. Rev Soc Bras Clin Med. 2015; 13(68):262-5. doi: http://files.bvs.br/upload/S/1679-1010/2015/v13n4/a5183.pdf

10. Sousa Júnior $A$ da S, Palácios VR da CM, Miranda C do S, Da Costa RJF, Catete CP, Chagasteles EJ, et al. Análise espaço-temporal da doença de chagas e seus fatores de risco ambientais e demográficos no município de Barcarena, Pará, Brasil. Rev Bras Epidemiol. 2017. 20(4):742-55. doi: http://files.bvs.br/upload/S/1679-1010/2015/ v13n4/a5183.pdf

11. Andrade NCDS De. Perfil Epidemiológico dos Pacientes Chagásicos atendidos em Hospital Público de Referência Cardiológica em
Belém/PA. Rev Cient Núcelo do Conhecimento. 2016. 9(1):118-35. Disponivel em: https://www.nucleodoconhecimento.com.br/saude/ pacientes-chagasicos-atendidos-em-hospital-publico?pdf $=4787$ Acesso em 24 ago 2019.

12. Pereira CML, Azevedo AP, Marinho S da SB, Prince KA de, Gonçalves JTT, Costa MR, et al. Perfil clínico e epidemiológico da doença de chagas aguda no estado de Minas Gerais. Rev Aten Saúde São Caetano do Sul. 2017.15(52):49-54. Disponivel em: http://seer. uscs.edu.br/index.php/revista_ciencias_saude/article/view/4523/ pdf Acesso em 22 ago 2019.

13. Filho JD da S, Costa AC, Freitas EC, Viana CEM, Lima MA, Andrade $\mathrm{MC}$, et al. Perfil hematológico e bioquímico de pacientes com doença de Chagas atendidos por um serviço de atenção farmacêutica no estado do Ceará TT. Hematological and biochemical profile of patients with Chagas disease attended by a pharmaceutical care service in. J Heal Biol Sci. 2017; 5(2):130-6. Disponivel em: http://periodicos. unichristus.edu.br/index.php/jhbs/article/view/1135 Acesso em 24 ago 2019.

14. Marques $A A$, Hennington ÉA. As repercussões da Doença de Chagas no contexto de vida e trabalho de usuários de instituto de pesquisa. Saúde em Debate. 2017; 41(spe2):21524. Doi: http://www.scielo.br/scielo.php?script=sci_arttext\&pid=S0103-11042017000600215\&lng=pt\&tlng=pt

15. Júnior $A S$ de $S$, Coelho HLL, Fonteles MM de $F$, Oliveira M de $F$. Problemas relacionados a medicamentos em pacientes com Doença de Chagas durante tratamento com benzonidazol em fortaleza Ce. Cad Saúde Colet. 2009;17(4):893-909. Disponivel em: http:// www.cadernos.iesc.ufrj.br/cadernos/images/csc/2009_4/artigos/ SeEspecial_8.pdf Acesso em 24 ago 2019.

16. Brasil. Ministério da Saúde. Protocolo Clínico e Diretrizes Terapêuticas Doença de Chagas. 1st ed. Brasília: Secretaria de Ciência, Tecnologia e Insumos Estratégicos. 2018; 141 p. Disponivel em: http://conitec.gov.br Acesso em: 24 ago 2019.

17. Spinelli JLM. et al. Análise espacial, clínico-epidemiológica e laboratorial de crianças internadas com leishmaniose visceral no Pará/ Amazônia Brasileira. Saúde Coletiva, 2021; (11)68 Doi: https://doi. org/10.36489/saudecoletiva.2021v11i68p7629-7646

18. Simões MV, Romano MMD, Schmidt A, Martins KSM, Marin-Neto JA. Cardiomiopatia da Doença de Chagas. Int J Cardiovasc Sci. 2018 acesso em 24 ago 2019; 31(2):173-89. Disponivel em: http://www. scielo.br/pdf/ijcs/v31n2/pt_2359-4802-ijcs-31-02-0173.pdf 